\title{
Ground-state entanglement in coupled qubits
}

\author{
A. Yu. Smirnov ${ }^{1}$ and M. H. Amin ${ }^{1,2}$ \\ ${ }^{1}$ D-Wave Systems Inc., 3033 Beta Avenue, Burnaby BC Canada V5G $4 M 9$ \\ ${ }^{2}$ Department of Physics, Simon Fraser University, Burnaby, BC Canada V5A $1 S 6$
}

\begin{abstract}
We study a system of qubits that are coupled to each other via only one degree of freedom represented, e.g., by $\sigma_{z}$-operators. We prove that, if by changing the Hamiltonian parameters, a non-degenerate ground state of the system is continuously transformed in such a way that the expectation values of $\sigma_{z}$ operators of at least two coupled qubits change, this ground state is entangled. Using this proof, we discuss connection between energy level anticrossings and ground state entanglement. Following the same line of thought, we introduce entanglement witnesses, based on cross-susceptibilities, that can detect ground state entanglement for any bipartition of the multiqubit system. A witness for global ground state entanglement is also introduced.
\end{abstract}

\section{INTRODUCTION}

Entanglement is considered to be an essential property required for quantum computation [1, 2]. It has been shown that a pure state quantum computation that does not possess some minimum level of entanglement can be efficiently simulated by classical computers [3, [4] .

For a pure state, entanglement can be defined in the following way [1]. Consider an arbitrary bipartition of the system $S$ into two parts $A$ and $B$, with Hilbert spaces $\mathcal{H}_{S}$ and $\mathcal{H}_{A, B}$, respectively. The system $S$ described by a wave function $|\Psi\rangle \in \mathcal{H}_{S}$ is entangled if there exists at least one bipartition $A$ and $B$ such that $|\Psi\rangle$ cannot be represented as a tensor product $\left|\Psi_{A}\right\rangle \otimes\left|\Psi_{B}\right\rangle$ of the states $\left|\Psi_{A}\right\rangle \in \mathcal{H}_{A}$ and $\left|\Psi_{B}\right\rangle \in \mathcal{H}_{B}$. By this definition, if an $N$ qubit system is not entangled, then it is completely separable: $|\Psi\rangle=\otimes_{i=1}^{N}\left|\psi_{i}\right\rangle$, where $\left|\psi_{i}\right\rangle$ is a single qubit wave function in the Hilbert space of qubit $i$. One can also define entanglement for a particular bipartition when $|\Psi\rangle$ is not separable relative to that partition. Global entanglement is defined when $|\Psi\rangle$ is not separable for any bipartition.

Entanglement is usually characterized by measures and witnesses [1, 5, 10]. A measure of entanglement provides a quantitative characterization for the amount of entanglement. A witness of entanglement, on the other hand, provides a sufficient but not necessary condition for entanglement. Usually, the condition is written in a form of an inequality. If the inequality is satisfied, the system is entangled, but if it does not, the system may or may not be entangled. Witnesses are constructed in such a way that they can be measured in practice [11, 12].

Experimentally, entanglement is commonly detected by quantum state tomography [13] or by measuring correlations between different parts of the system that are not allowed by classical physics [14, 15]. Most experiments involve preparing the system in a particular state by turning on interactions between the subsystems, but performing measurements when there is no interaction between the qubits. This is different from detecting entanglement when the interaction between the subsystems is always present. Detecting entanglement in that case, for example, when the system is in an eigenstate of a
Hamiltonian, requires a different approach. Any such approach is inherently restricted by experimental limitations: the ability to measure qubits in a particular basis, limitations in time domain control, etc.

Spectroscopy is one of the tools that has been used to detect coherence and entanglement in multi-qubit systems [16 18]. In these measurements, observation of an anticrossing between two energy levels is taken to be an evidence for eigenstate superposition or entanglement. While this is intuitively clear, to our knowledge there is no mathematical justification to support it. In this paper, we prove a theorem that relates anticrossings with ground state entanglement for a system of qubits with pairwise coupling via a single degree of freedom.

Magnetic susceptibility has also been used to detect entanglement 19]. An entanglement witness using a sum of susceptibilities taken along $x$-, $y$-, and $z$-directions has been proposed in Ref. [20] and measured in Refs. 21, 22]. The witness requires measurements of average values of spin projections in all three dimensions. This becomes problematic when only one component of the spin projection can be measured, unless the system is isotropic [23]. Here, we introduce witnesses, based on only one component of the susceptibility, to detect ground state entanglement. No detailed knowledge of the Hamiltonian, except that the qubits are pairwise coupled via $\sigma_{z^{-}}$ operators, is needed. These witnesses can provide useful tools for experimental demonstration of entanglement in a quantum annealing processor 24,25$]$.

The paper is organized as follows. In Sec. II we prove that anticrossing of energy levels can be considered as a signature of entanglement. Susceptibility-based entanglement witnesses are described in Sec. III and conclusions are provided in Sec. IV.

\section{ANTICROSSINGS AND ENTANGLEMENT}

Observation of avoided crossings or anticrossings between two energy levels have been used as evidence for coherent superposition [16] and entanglement [17, 26]. It is intuitively evident that at the center of an avoided crossing the eigenstates of the many-qubit system are su- 
perpositions of the two crossing states and therefore can be entangled. If all terms in the Hamiltonian are known, one can easily check by exact diagonalization whether or not the eigenstates are entangled. But without the exact knowledge of the Hamiltonian, is it possible to conclude anything about the entanglement?

Consider a system of qubits described by the following Hamiltonian

$$
H=\sum_{i=1}^{N} H_{i}+\frac{1}{2} \sum_{i, j=1}^{N} J_{i j} \sigma_{i}^{z} \sigma_{j}^{z},
$$

where $N$ is the number of qubits, $H_{i}$ is a single qubit Hamiltonian acting on the $i$-th qubit, $J_{j i}=J_{i j}$, and $J_{i i}=0$. Operators $\sigma_{i}^{z}$ acting in the many-qubit Hilbert space are defined as

$$
\sigma_{i}^{z} \equiv \otimes_{k=1}^{N} \hat{o}_{k}, \quad \hat{o}_{k}=\left\{\begin{array}{cl}
\sigma_{z} & \text { if } k=i \\
I & \text { if } k \neq i
\end{array}\right.
$$

where $I$ is a $2 \times 2$ identity matrix and $\sigma_{z}$ is a standard Pauli matrix, $\sigma_{z}=\left(\begin{array}{cc}1 & 0 \\ 0 & -1\end{array}\right)$. We assume no knowledge of $H_{i}$ and the exact values of $J_{i j}$. The only knowledge is that the qubits are coupled via $\sigma_{i}^{z}$-operators. In general, $H$ is a function of a set of external parameters which we collectively denote by $\lambda$. These parameters are, for example, external voltages, currents, or fluxes applied to the physical qubits.

Let $\left|\Psi_{0}(\lambda)\right\rangle$ be the ground state of $H(\lambda)$, and $\left\langle\sigma_{j}^{z}\right\rangle \equiv\left\langle\Psi_{0}\left|\sigma_{j}^{z}\right| \Psi_{0}\right\rangle$.

Theorem: Consider Hamiltonian (1) that has at least one nonzero coupling and is a continuous function of $\lambda \in \Lambda$, where $\Lambda$ is a connected region in the parameter space. If $\left|\Psi_{0}(\lambda)\right\rangle$ is a completely separable non-degenerate ground state of $H(\lambda)$, then for every pair $\{i, j\}$ of qubits with $J_{i j} \neq 0$, there is at least one average spin projection, $\left\langle\sigma_{i}^{z}\right\rangle$ or $\left\langle\sigma_{j}^{z}\right\rangle$ or both, which does not change with $\lambda$.

We provide a proof for this theorem in appendix A. Here, we only focus on its implications. The theorem states that if there is at least one pair of coupled qubits $i$ and $j$ (with $J_{i j} \neq 0$ ) for which the expectation values $\left\langle\sigma_{i}^{z}\right\rangle$ and $\left\langle\sigma_{j}^{z}\right\rangle$ of both qubits simultaneously change when the Hamiltonian parameters change, and if the system stays in a unique eigenstate during this change, then that eigenstate should be entangled.

The best example is when the ground state of the system goes through an anticrossing as the parameters are varied. Consider the transverse Ising Hamiltonian

$$
H_{\mathrm{TI}}=-\frac{1}{2} \sum_{i} \Delta_{i} \sigma_{i}^{x}-\sum_{i} h_{i} \sigma_{i}^{z}+\sum_{i<j} J_{i j} \sigma_{i}^{z} \sigma_{j}^{z},
$$

where $\sigma_{i}^{x}$ are defined similar to $\sigma_{i}^{z}$ in (2). We focus on a system of ferromagnetically coupled qubits with $J_{i j}=-J$, all subject to a uniform energy bias $h_{i}=h$.
When $h=-h_{0}<0$ is a large negative number, the state of the system will be close to the ferromagnetically ordered state $|\downarrow \downarrow \ldots \downarrow\rangle$. On the other hand for large positive values, $h=h_{0}>0$, the state of the system will be close to $|\uparrow \uparrow \ldots \uparrow\rangle$. In this example, $h$ plays the role of $\lambda$. Clearly at $\lambda_{\text {in }}=h_{\text {in }}=-h_{0}$, all qubits have $\left\langle\sigma_{i}^{z}\left(\lambda_{\text {in }}\right)\right\rangle \approx-1$ in the ground state and at $\lambda_{\text {fin }}=h_{\text {fin }}=h_{0}$, all qubits have $\left\langle\sigma_{i}^{z}\left(\lambda_{\text {fin }}\right)\right\rangle \approx 1$. Therefore the ground state expectation value of $\sigma_{i}^{z}$ changes for all qubits. Now, if in changing from $\lambda_{\text {in }}$ to $\lambda_{\text {fin }}$ the system goes through an anticrossing, it means that one can continuously change the ground state of the system without going through any degeneracy, i.e., the ground state remains non-degenerate all through the change. In that case, the above theorem states that the ground state of the system has to go through an entangled state during this evolution unless all qubits are uncoupled $\left(J_{i j}=0, \forall i, j\right)$. The latter can be easily checked if instead of applying a uniform bias to all qubits, one applies a bias to only one of the qubits. In that case, if the qubits are uncoupled, only $\left\langle\sigma_{i}^{z}\right\rangle$ of the qubit to which the bias is applied will change and the other qubits remain unaffected. Therefore, if by applying a bias to one qubit, the expectation value $\left\langle\sigma_{i}^{z}\right\rangle$ for all other qubits get affected, then the qubits should be coupled. Observation of such an anticrossing, therefore, is evidence for the existence of entangled ground state. Notice that knowledge of the exact values of $J_{i j}$ or any other Hamiltonian parameters is not necessary to prove ground state entanglement.

\section{SUSCEPTIBILITY-BASED ENTANGLEMENT WITNESSES}

The theorem in the previous section suggests a close relation between entanglement and susceptibility. Let us define susceptibility of qubit $i$ to $\lambda$ as

$$
\chi_{i}^{\lambda}=\partial\left\langle\sigma_{i}^{z}\right\rangle / \partial \lambda
$$

The theorem states that for two coupled qubits, $J_{i j} \neq 0$, if both susceptibilities $\chi_{i}^{\lambda}$ and $\chi_{j}^{\lambda}$ are nonzero, then the state is entangled. This suggests introducing

$$
\mathcal{W}_{\lambda}=\sum_{i j}\left|J_{i j} \chi_{i}^{\lambda} \chi_{j}^{\lambda}\right|
$$

which is zero if the state is completely separable. A nonzero value of $\mathcal{W}_{\lambda}$ means that at least for one pair of qubits, all three $J_{i j}, \chi_{i}^{\lambda}$, and $\chi_{j}^{\lambda}$ are nonzero and therefore the system is entangled. The inequality $\mathcal{W}_{\lambda}>0$, therefore, is a sufficient condition for entanglement. As such, we consider $\mathcal{W}_{\lambda}$ as an entanglement witness, based on our convention. The above witness, however, cannot determine how many qubits are entangled to each other. Even if only two qubits are entangled, the witness will be nonzero.

One can generalize the above idea to introduce witnesses that can distinguish any bipartite entanglement. 
For the rest of this section we focus on the transverse Ising Hamiltonian (3), for which we can define the ground state cross-susceptibility between qubit $i$ and qubit $j$ as

$$
\chi_{i j}=\frac{\partial\left\langle\sigma_{i}^{z}\right\rangle}{\partial h_{j}}
$$

For a Hamiltonian $H$ with eigenstates $\left|\Psi_{n}\right\rangle$ and eigenvalues $E_{n}$, the susceptibility can be written as (see Ref. [27] and also Appendix B)

$$
\chi_{i j}=\sum_{n>0} \frac{\left\langle\Psi_{0}\left|\sigma_{j}^{z}\right| \Psi_{n}\right\rangle\left\langle\Psi_{n}\left|\sigma_{i}^{z}\right| \Psi_{0}\right\rangle}{E_{n}-E_{0}}+c . c .
$$

Here, c.c. means the complex conjugate of the previous expression.

Consider an arbitrary bipartition of the system $S$ into two parts $A$ and $B, S=A \cup B$. Recall that these two parts are separable if the wave function of $S$ can be written as $\left|\Psi_{0}\right\rangle=\left|\Psi_{0}^{A}\right\rangle \otimes\left|\Psi_{0}^{B}\right\rangle$, otherwise, they are entangled. Our first goal is to define a witness that can detect entanglement between $A$ and $B$ when $\left|\Psi_{0}\right\rangle$ is a non-degenerate ground state of $H$. Let us introduce

$$
\widetilde{\mathcal{W}}_{A B}=\sum_{i \in A, j \in B} J_{i j} \chi_{i j}
$$

Using (7), we can write

$$
\begin{aligned}
& \widetilde{\mathcal{W}}_{A B}=\sum_{n>0} \frac{1}{E_{n}-E_{0}} \\
& \times \sum_{i \in A, j \in B} J_{i j}\left\langle\Psi_{0}\left|\sigma_{j}^{z}\right| \Psi_{n}\right\rangle\left\langle\Psi_{n}\left|\sigma_{i}^{z}\right| \Psi_{0}\right\rangle+\text { c.c. }
\end{aligned}
$$

It is clear that $\widetilde{\mathcal{W}}_{A B}$ is not only a function of the ground state, $\left|\Psi_{0}\right\rangle$, but also a function of all other eigenstates. However, as we shall see, it can still be used to detect the ground state entanglement.

Let us assume that the ground state is separable, $\left|\Psi_{0}\right\rangle=\left|\Psi_{0}^{A}\right\rangle \otimes\left|\Psi_{0}^{B}\right\rangle$. We introduce two reduced vectors,

$$
\left|\widetilde{\Psi}_{n}^{A}\right\rangle=\left\langle\Psi_{0}^{B} \mid \Psi_{n}\right\rangle \in \mathcal{H}_{A}, \quad\left|\widetilde{\Psi}_{n}^{B}\right\rangle=\left\langle\Psi_{0}^{A} \mid \Psi_{n}\right\rangle \in \mathcal{H}_{B} .
$$

Due to orthogonality of the ground and excited states,

$$
\left\langle\widetilde{\Psi}_{n}^{A} \mid \Psi_{0}^{A}\right\rangle=0, \quad\left\langle\widetilde{\Psi}_{n}^{B} \mid \Psi_{0}^{B}\right\rangle=0
$$

By definition, $\left|\Psi_{0}\right\rangle$ is an eigenstate of (3), thus

$$
\left(H_{A}+H_{B}+\sum_{i \in A, j \in B} J_{i j} \sigma_{i}^{z} \sigma_{j}^{z}\right)\left|\Psi_{0}\right\rangle=E_{0}\left|\Psi_{0}\right\rangle .
$$

Here, $H_{A}$ and $H_{B}$ describe the subsystems $A$ and $B$, respectively. We can multiply Eq. (11) by $\left\langle\widetilde{\Psi}_{n}^{B}\right|$ to obtain

$$
\sum_{i \in A, j \in B} J_{i j}\left\langle\widetilde{\Psi}_{n}^{B}\left|\sigma_{j}^{z}\right| \Psi_{0}^{B}\right\rangle \sigma_{i}^{z}\left|\Psi_{0}^{A}\right\rangle=-\left\langle\widetilde{\Psi}_{n}^{B}\left|H_{B}\right| \Psi_{0}^{B}\right\rangle\left|\Psi_{0}^{A}\right\rangle .
$$

Multiplying by $\otimes\left|\Psi_{0}^{B}\right\rangle$ and using the definition of $\left|\widetilde{\Psi}_{n}^{B}\right\rangle$, we find

$$
\sum_{i \in A, j \in B} J_{i j}\left\langle\Psi_{n}\left|\sigma_{j}^{z}\right| \Psi_{0}\right\rangle \sigma_{i}^{z}\left|\Psi_{0}\right\rangle=-\left\langle\widetilde{\Psi}_{n}^{B}\left|H_{B}\right| \Psi_{0}^{B}\right\rangle\left|\Psi_{0}\right\rangle .
$$

Taking complex conjugate of all terms we get

$$
\sum_{i \in A, j \in B} J_{i j}\left\langle\Psi_{0}\left|\sigma_{j}^{z}\right| \Psi_{n}\right\rangle \sigma_{i}^{z}\left|\Psi_{0}\right\rangle^{*}=-\left\langle\Psi_{0}^{B}\left|H_{B}\right| \widetilde{\Psi}_{n}^{B}\right\rangle\left|\Psi_{0}\right\rangle^{*},
$$

where $\left|\Psi_{0}\right\rangle^{*}$ is a vector with each component being the complex conjugate of the corresponding component in $\left|\Psi_{0}\right\rangle$. If the Hamiltonian is real in the computation basis, $H=H^{*}$, i.e., only contains $\sigma_{i}^{x}$ and $\sigma_{i}^{z}$ matrices with real coefficients, then $H\left|\Psi_{0}\right\rangle^{*}=E_{0}\left|\Psi_{0}\right\rangle^{*}$. Therefore, $\left|\Psi_{0}\right\rangle$ and $\left|\Psi_{0}\right\rangle^{*}$ should be proportional to each other, $\left|\Psi_{0}\right\rangle^{*}=e^{i \varphi_{0}}\left|\Psi_{0}\right\rangle$, (up to a constant phase factor $\left.e^{i \varphi_{0}}\right)$ provided that the ground energy level $E_{0}$ is non-degenerate. Replacing $\left|\Psi_{0}\right\rangle^{*}$ with $\left|\Psi_{0}\right\rangle$ and applying $\left\langle\Psi_{n}\right|$ from the left, we get

$$
\sum_{i \in A, j \in B} J_{i j}\left\langle\Psi_{0}\left|\sigma_{j}^{z}\right| \Psi_{n}\right\rangle\left\langle\Psi_{n}\left|\sigma_{i}^{z}\right| \Psi_{0}\right\rangle=0 .
$$

Comparing with the second line of (9), we find $\widetilde{\mathcal{W}}_{A B}=0$. Therefore, if the measurements show that $\widetilde{\mathcal{W}}_{A B} \neq 0$, this means that $\left|\Psi_{0}\right\rangle$ is not separable relative to $A$ and $B$ and therefore is entangled. This is true independent of whether the excited states are separable or entangled.

Since susceptibility can be divergent, $\widetilde{\mathcal{W}}_{A B}$ is not bounded. To have a witness that is bounded by 1 and is independent of the number of the connections between the subsystems $A$ and $B$, it is useful to introduce

$$
\mathcal{W}_{A B}=\frac{\left|\widetilde{\mathcal{W}}_{A B}\right|}{N_{A B}+\left|\widetilde{\mathcal{W}}_{A B}\right|},
$$

where $N_{A B}$ is the number of non-zero couplings between subsystems $A$ and $B$. From the above argument, a nonzero value of $\mathcal{W}_{A B}$ means that the two parts $A$ and $B$ are entangled in the ground state of the transverse Ising Hamiltonian.

The witness $\mathcal{W}_{A B}$ can only determine entanglement between $A$ and $B$. It cannot determine whether every part of $S$ is entangled with every other part. To detect the global entanglement, one can use geometric averages 9]. Suppose that there are $N_{p}$ possible bipartitions of $S$. A global entanglement witness can be defined as

$$
\mathcal{W}_{\chi}=\frac{\left[\prod\left(\widetilde{\mathcal{W}}_{A B} / N_{A B}\right)\right]^{1 / N_{p}}}{1+\left[\prod\left(\widetilde{\mathcal{W}}_{A B} / N_{A B}\right)\right]^{1 / N_{p}}}
$$

where $\prod$ denotes the product over all $N_{P}$ possible bipartitions of $S$. Similar to (13), $\mathcal{W}_{\chi}$ is bounded by 1 . A nonzero value of $\mathcal{W}_{\chi}$ means that all parts of $S$ are entangled to each other, therefore, the system is globally entangled. 


\section{CONCLUSIONS}

Ground state entanglement in a system of qubits that are pairwise coupled to each other via a single degree of freedom has been studied. We have presented a theorem that relates eigenstate entanglement with anticrossing of the energy levels. We have introduced ground state entanglement witnesses, based on susceptibility, which can detect any bipartite entanglement as well as the global entanglement. This approach enables experimental demonstration of entanglement of coupled qubits without detailed information of the system Hamiltonian.

In order to apply these results in experiments, one needs to have access to spectroscopy, a method to measure ground state average of the spin projection operator, $\left\langle\sigma_{i}^{z}\right\rangle$, and a way to measure coupling strengths or at least determine if they are nonzero. Information about the dependence of $\left\langle\sigma_{i}^{z}\right\rangle$ on the Hamiltonian parameters is important for the proof of entanglement via anticrossings as well as for the extraction of the susceptibilities used in the entanglement witnesses. Usually, in experiments $\sigma_{i}^{z}$ is measured in the thermal state. To assure that the measurement provides a good approximation for the ground state average $\left\langle\sigma_{i}^{z}\right\rangle$, it is important to have a pronounced energy gap, in the spectroscopic data, between the ground and first excited states. The gap has to be larger than the energy level broadening and temperature to guarantee that the ground state remains the only thermally occupied state during the measurement.

\section{Acknowledgements}

We are grateful to S. Boixo, F. Spedalieri, T. Lanting, and P. Love for helpful discussions.

\section{Appendix A: Proof of the theorem}

In this appendix, we provide a proof for the theorem presented in Sec. II. First, we prove a few Lemmas. Consider Hamiltonian $H$ defined by (1).

Lemma 1: If $H$ has a completely separable eigenstate $|\Psi\rangle=\otimes_{i=1}^{N}\left|\psi_{i}\right\rangle$, where $\left|\psi_{i}\right\rangle$ is a state in the Hilbert space of the $i$-th qubit, then for each nonzero coupler, $J_{i j} \neq 0$, either $\left|\psi_{i}\right\rangle$ or $\left|\psi_{j}\right\rangle$ is an eigenfunction of the $\sigma_{z}$-operator: $\sigma_{z}\left|\psi_{i}\right\rangle=z_{i}\left|\psi_{i}\right\rangle$ or $\sigma_{z}\left|\psi_{j}\right\rangle=z_{j}\left|\psi_{j}\right\rangle$, with corresponding eigenvalues $z_{i}$ or $z_{j}\left(z_{i} \in\{0,1\}\right)$.

If $|\Psi\rangle$ is an eigenstate of $H$, then

$$
H|\Psi\rangle=E|\Psi\rangle
$$

We multiply both sides of (A1) by $\left(\otimes_{k \neq i, j}\left\langle\psi_{k}\right|\right) \otimes\left\langle\widetilde{\psi}_{j}\right|$, where $\left|\widetilde{\psi}_{j}\right\rangle$ is a state orthogonal to $\left|\psi_{j}\right\rangle:\left\langle\widetilde{\psi}_{j} \mid \psi_{j}\right\rangle=0$. Using (11) we find

$$
J_{i j}\left\langle\widetilde{\psi}_{j}\left|\sigma_{z}\right| \psi_{j}\right\rangle \sigma_{z}\left|\psi_{i}\right\rangle=E_{i}\left(\widetilde{\psi}_{j}\right)\left|\psi_{i}\right\rangle
$$

where

$$
\begin{aligned}
E_{i}\left(\widetilde{\psi}_{j}\right)= & -\left\langle\widetilde{\psi}_{j}\left|H_{j}\right| \psi_{j}\right\rangle \\
& -\sum_{k \neq i, j} J_{k j}\left\langle\psi_{k}\left|\sigma_{z}\right| \psi_{k}\right\rangle\left\langle\widetilde{\psi}_{j}\left|\sigma_{z}\right| \psi_{j}\right\rangle
\end{aligned}
$$

Since $J_{i j} \neq 0$, Eq. A2 requires $\left|\psi_{i}\right\rangle$ to be an eigenfunction of $\sigma_{z}$ unless if $\left\langle\widetilde{\psi}_{j}\left|\sigma_{z}\right| \psi_{j}\right\rangle=0$. The latter, however, requires $\left|\psi_{j}\right\rangle$ to be an eigenfunction of $\sigma_{z}$. To see this, we notice that the orthogonal set $\left\{\left|\psi_{j}\right\rangle,\left|\widetilde{\psi}_{j}\right\rangle\right\}$ forms a complete set of bases in the Hilbert space of qubit $j$. One can therefore write $\sigma_{z}\left|\psi_{j}\right\rangle=c_{1}\left|\psi_{j}\right\rangle+c_{2}\left|\widetilde{\psi}_{j}\right\rangle$. If $\left\langle\widetilde{\psi}_{j}\left|\sigma_{z}\right| \psi_{j}\right\rangle=0$, then $c_{2}=0$ and therefore $\left|\psi_{j}\right\rangle$ should be an eigenfunction of $\sigma_{z}$ with eigenvalue $c_{1}(= \pm 1)$. This means that at least one of $\left|\psi_{i}\right\rangle$ and $\left|\psi_{j}\right\rangle$ is an eigenfunction of $\sigma_{z}$.

From (A2) and A3 , it also follows that if $\left|\psi_{j}\right\rangle$ is an eigenfunction of $\sigma_{z}$, then $E_{i}\left(\widetilde{\psi}_{j}\right)=-\left\langle\widetilde{\psi}_{j}\left|H_{j}\right| \psi_{j}\right\rangle=0$. Therefore, $H_{j}$ should also be diagonal in the basis $\left\{\left|\psi_{j}\right\rangle,\left|\widetilde{\psi}_{j}\right\rangle\right\}$.

Lemma 2: Consider a state $|\Psi\rangle=\left|z_{i}\right\rangle \otimes\left|\Phi^{i}\right\rangle$, where $\left|z_{i}\right\rangle$ is an eigenfunction of $\sigma_{z}\left(z_{i} \in\{0,1\}\right)$ representing the state of qubit $i$, and $\left|\Phi^{i}\right\rangle$ is a state in the Hilbert space, $S_{i}$, of all qubits except the ith one. If $|\Psi\rangle$ is an eigenstate of Hamiltonian (1), then all eigenstates of $H$ can be represented as either $\left|\Psi_{n}\right\rangle=\left|z_{i}\right\rangle \otimes\left|\Phi_{n}^{i}\right\rangle$ or $\left|\bar{\Psi}_{n}\right\rangle=\left|\bar{z}_{i}\right\rangle \otimes\left|\bar{\Phi}_{n}^{i}\right\rangle$, where $\bar{z}_{i}=1-z_{i}$, and $\left\{\left|\Phi_{n}^{i}\right\rangle\right\}$ and $\left\{\left|\bar{\Phi}_{n}^{i}\right\rangle\right\}$, with $n=1, \ldots, 2^{N-1}$, are two independent complete orthogonal sets in $S_{i}$.

Multiplying both sides of (A1) by $\langle\Phi| \otimes\left\langle\bar{z}_{i}\right|$ and using $\left\langle\bar{z}_{i}\left|\sigma_{z}\right| z_{i}\right\rangle=0$, we find $\left\langle\bar{z}_{i}\left|H_{i}\right| z_{i}\right\rangle=0$. This means that $H_{i}$ is diagonal in the basis $\{|0\rangle,|1\rangle\}$, i.e., $H_{i}\left|z_{i}\right\rangle=E_{i}\left|z_{i}\right\rangle$ and $H_{i}\left|\bar{z}_{i}\right\rangle=\bar{E}_{i}\left|\bar{z}_{i}\right\rangle$. Defining $\left|\Psi_{n}\right\rangle=\left|z_{i}\right\rangle \otimes\left|\Phi_{n}^{i}\right\rangle$ and $\left|\bar{\Psi}_{n}\right\rangle=\left|\bar{z}_{i}\right\rangle \otimes\left|\bar{\Phi}_{n}^{i}\right\rangle$, with $n=1, \ldots, 2^{N-1}$, and using $\sigma_{z}\left|z_{i}\right\rangle=\alpha_{i}\left|z_{i}\right\rangle$, where $\alpha_{i} \equiv 2 z_{i}-1(= \pm 1)$, we find

$$
\begin{aligned}
H\left|\Psi_{n}\right\rangle & =\left|z_{i}\right\rangle \otimes H_{\Phi}^{i}\left|\Phi_{n}^{i}\right\rangle, \\
H\left|\bar{\Psi}_{n}\right\rangle & =\left|\bar{z}_{i}\right\rangle \otimes \bar{H}_{\Phi}^{i}\left|\bar{\Phi}_{n}^{i}\right\rangle,
\end{aligned}
$$

where

$$
\begin{aligned}
& H_{\Phi}^{i}=E_{i}+\sum_{j \neq i}\left(H_{j}+\alpha_{i} J_{i j} \sigma_{j}^{z}\right)+\frac{1}{2} \sum_{j, k \neq i} J_{j k} \sigma_{k}^{z} \sigma_{j}^{z} \\
& \bar{H}_{\Phi}^{i}=\bar{E}_{i}+\sum_{j \neq i}\left(H_{j}-\alpha_{i} J_{i j} \sigma_{j}^{z}\right)+\frac{1}{2} \sum_{j, k \neq i} J_{j k} \sigma_{k}^{z} \sigma_{j}^{z} .
\end{aligned}
$$

From (A4), it is clear that if $\left|\Phi_{n}^{i}\right\rangle$ is an eigenfunction of $H_{\Phi}^{i}$ with eigenvalue $E_{n}$ and $\left|\bar{\Phi}_{n}^{i}\right\rangle$ is an eigenfunction of $\bar{H}_{\Phi}^{i}$ with eigenvalue $\bar{E}_{n}$, then $H\left|\Psi_{n}\right\rangle=E_{n}\left|\Psi_{n}\right\rangle$ and $H\left|\bar{\Psi}_{n}\right\rangle=\bar{E}_{n}\left|\bar{\Psi}_{n}\right\rangle$. Since $\left\{\left|\Psi_{n}\right\rangle\right\}$ and $\left\{\left|\bar{\Psi}_{n}\right\rangle\right\}$ are orthogonal to each other, and there are $2^{N-1}$ states in each set, all $2^{N}$ eigenstates of $H$ are in these two sets.

Proof of the theorem: Assume that $H(\lambda)$ has a completely separable ground state $\left|\Psi_{0}(\lambda)\right\rangle=\otimes_{k=1}^{N}\left|\psi_{k}(\lambda)\right\rangle$. 
We consider a pair $\{i, j\}$ of connected qubits with $J_{i j} \neq 0$. It follows from Lemma 1 that at least one of the two states, $\left|\psi_{i}\right\rangle$ or $\left|\psi_{j}\right\rangle$, should be an eigenstate of the $\sigma_{z}$-matrix. We consider two points, $\lambda_{L}=\lambda_{0}-\delta \lambda / 2$ and $\lambda_{R}=\lambda_{0}+\delta \lambda / 2$, located in the close proximity of an arbitrary point $\lambda_{0} \in \Lambda$. Without loss of generality, we take $\left|\psi_{i}\right\rangle$ be a $\sigma_{z}$-eigenstate at $\lambda_{L}:\left|\psi_{i}\left(\lambda_{L}\right)\right\rangle=\left|z_{i}\right\rangle$. Based on Lemma 1, either $\left|\psi_{i}\left(\lambda_{R}\right)\right\rangle$ or $\left|\psi_{j}\left(\lambda_{R}\right)\right\rangle$ should be a $\sigma_{z}$-eigenstate. There are three possibilities:

(i) $\left|\psi_{i}\left(\lambda_{R}\right)\right\rangle=\left|z_{i}\right\rangle$. Thus, the direction of the $i$-th spin does not change during the infinitesimal transition from $\lambda_{L}$ to $\lambda_{R}:\left\langle\sigma_{i}^{z}\right\rangle_{\lambda=\lambda_{L}}=\left\langle\sigma_{i}^{z}\right\rangle_{\lambda=\lambda_{R}}=z_{i}$.

(ii) $\left|\psi_{i}\left(\lambda_{R}\right)\right\rangle=\left|\bar{z}_{i}\right\rangle$ with $\bar{z}_{i}=1-z_{i}$. The flipping of the $i$ th qubit between $\lambda_{L}$ and $\lambda_{R}$ requires a crossing of energy levels, in contradiction to the theorem conditions. To see this, let us write

$$
\begin{aligned}
\left|\Psi_{0}\left(\lambda_{L}\right)\right\rangle & =\left|z_{i}\right\rangle \otimes\left|\Phi^{i}\left(\lambda_{L}\right)\right\rangle, \\
\left|\bar{\Psi}_{0}\left(\lambda_{R}\right)\right\rangle & =\left|\bar{z}_{i}\right\rangle \otimes\left|\bar{\Phi}^{i}\left(\lambda_{R}\right)\right\rangle,
\end{aligned}
$$

where $\left|\Phi^{i}\left(\lambda_{L}\right)\right\rangle$ and $\left|\bar{\Phi}^{i}\left(\lambda_{R}\right)\right\rangle$ are eigenstates of $H_{\Phi}^{i}\left(\lambda_{L}\right)$ and $\bar{H}_{\Phi}^{i}\left(\lambda_{R}\right)$ defined in (A5) and (A6):

$$
\begin{aligned}
& H_{\Phi}^{i}\left(\lambda_{L}\right)\left|\Phi^{i}\left(\lambda_{L}\right)\right\rangle=E_{0}\left(\lambda_{L}\right)\left|\Phi^{i}\left(\lambda_{L}\right)\right\rangle \\
& \bar{H}_{\Phi}^{i}\left(\lambda_{R}\right)\left|\Phi^{i}\left(\lambda_{R}\right)\right\rangle=\bar{E}_{0}\left(\lambda_{R}\right)\left|\bar{\Phi}^{i}\left(\lambda_{R}\right)\right\rangle .
\end{aligned}
$$

Since (A7) are the ground states of (11) at the two points, we have

$$
\begin{aligned}
& H\left|\Psi_{0}\left(\lambda_{L}\right)\right\rangle=E_{0}\left(\lambda_{L}\right)\left|\Psi_{0}\left(\lambda_{L}\right)\right\rangle, \\
& H\left|\bar{\Psi}_{0}\left(\lambda_{R}\right)\right\rangle=\bar{E}_{0}\left(\lambda_{R}\right)\left|\Psi_{0}\left(\lambda_{R}\right)\right\rangle .
\end{aligned}
$$

According to Lemma 2 the following states,

$$
\begin{aligned}
\left|\bar{\Psi}_{e}\left(\lambda_{L}\right)\right\rangle & =\left|\bar{z}_{i}\right\rangle \otimes\left|\bar{\Phi}^{i}\left(\lambda_{L}\right)\right\rangle, \\
\left|\Psi_{e}\left(\lambda_{R}\right)\right\rangle & =\left|z_{i}\right\rangle \otimes\left|\Phi^{i}\left(\lambda_{R}\right)\right\rangle,
\end{aligned}
$$

describe excited eigenstates of the Hamiltonian $H$,

$$
\begin{aligned}
& H\left|\bar{\Psi}_{e}\left(\lambda_{L}\right)\right\rangle=\bar{E}_{e}\left(\lambda_{L}\right)\left|\Psi_{e}\left(\lambda_{L}\right)\right\rangle, \\
& H\left|\Psi_{e}\left(\lambda_{R}\right)\right\rangle=E_{e}\left(\lambda_{R}\right)\left|\Psi_{e}\left(\lambda_{R}\right)\right\rangle,
\end{aligned}
$$

with eigenenergies $\bar{E}_{e}\left(\lambda_{L}\right) \geq E_{0}\left(\lambda_{L}\right)$ and $E_{e}\left(\lambda_{R}\right) \geq$ $\bar{E}_{0}\left(\lambda_{R}\right)$, which can be found from the equations

$$
\begin{aligned}
& \bar{H}_{\Phi}^{i}\left(\lambda_{L}\right)\left|\bar{\Phi}^{i}\left(\lambda_{L}\right)\right\rangle=\bar{E}_{e}\left(\lambda_{L}\right)\left|\bar{\Phi}^{i}\left(\lambda_{L}\right)\right\rangle, \\
& H_{\Phi}^{i}\left(\lambda_{R}\right)\left|\Phi^{i}\left(\lambda_{R}\right)\right\rangle=E_{e}\left(\lambda_{R}\right)\left|\Phi^{i}\left(\lambda_{R}\right)\right\rangle .
\end{aligned}
$$

Since $H$ is a continuous function of $\lambda$ by definition, $H_{\Phi}^{i}$ and $\bar{H}_{\Phi}^{i}$ should also be continuous functions. Thus, as $\lambda_{L} \rightarrow \lambda_{R}(\delta \lambda \rightarrow 0)$ we have: $H_{\Phi}^{i}\left(\lambda_{L}\right) \rightarrow H_{\Phi}^{i}\left(\lambda_{R}\right)$ and $\bar{H}_{\Phi}^{i}\left(\lambda_{L}\right) \rightarrow \bar{H}_{\Phi}^{i}\left(\lambda_{R}\right)$. This means that $\left|\Phi^{i}\left(\lambda_{L}\right)\right\rangle \rightarrow$ $\left|\Phi^{i}\left(\lambda_{R}\right)\right\rangle$ and $\left|\bar{\Phi}^{i}\left(\lambda_{L}\right)\right\rangle \rightarrow\left|\bar{\Phi}^{i}\left(\lambda_{R}\right)\right\rangle$. Comparing the first line of (A8) and the second line of (A12), and vice versa. we find that at $\lambda_{L}=\lambda_{R}=\lambda_{0}$,

$$
E_{0}\left(\lambda_{0}\right)=E_{e}\left(\lambda_{0}\right), \quad \bar{E}_{0}\left(\lambda_{0}\right)=\bar{E}_{e}\left(\lambda_{0}\right) .
$$

Since $E_{0}\left(\lambda_{0}\right)$ and $\bar{E}_{0}\left(\lambda_{0}\right)$ both represent the ground state energy at $\lambda_{0}$, they should be equal, therefore,

$$
E_{0}\left(\lambda_{0}\right)=\bar{E}_{e}\left(\lambda_{0}\right)=\bar{E}_{0}\left(\lambda_{0}\right)=E_{e}\left(\lambda_{0}\right)
$$

We note that the energy levels $E_{0}$ and $\bar{E}_{0}$ correspond to the eigenstates $\left|\Psi_{0}\right\rangle$ and $\left|\bar{\Psi}_{0}\right\rangle$ distinguished by opposite directions of the $i$ th spin (see Eqs. (A7)). This means that there needs to be a degeneracy at $\lambda=\lambda_{0}$, in contradiction with the original assumptions.

(iii) $\left|\psi_{j}\left(\lambda_{R}\right)\right\rangle=\left|z_{j}\right\rangle$. The ground states at $\lambda=\lambda_{L}$ and $\lambda=\lambda_{R}$ are determined by

$$
\begin{aligned}
\left|\Psi_{0}\left(\lambda_{L}\right)\right\rangle & =\left|z_{i}\right\rangle \otimes\left|\Phi^{i}\left(\lambda_{L}\right)\right\rangle, \\
\left|\Psi_{0}\left(\lambda_{R}\right)\right\rangle & =\left|z_{j}\right\rangle \otimes\left|\Phi^{j}\left(\lambda_{R}\right)\right\rangle .
\end{aligned}
$$

Since $H(\lambda)$ is continuous, one can use Taylor expansion

$$
H\left(\lambda_{L}\right)=H\left(\lambda_{R}\right)-\left[\frac{\delta H(\lambda)}{\delta \lambda}\right]_{\lambda=\lambda_{R}} \delta \lambda .
$$

Applying the perturbation theory we find the ground state function at $\lambda=\lambda_{R}$,

$$
\left|\Psi_{0}\left(\lambda_{L}\right)\right\rangle=\left|\Psi_{0}\left(\lambda_{R}\right)\right\rangle-\delta \lambda\left|\Psi_{0}^{\prime}\left(\lambda_{R}\right)\right\rangle,
$$

where

$$
\left|\Psi_{0}^{\prime}(\lambda)\right\rangle=\sum_{n>0} \frac{\left\langle\Psi_{n}(\lambda)\left|\frac{\delta H(\lambda)}{\delta \lambda}\right| \Psi_{0}(\lambda)\right\rangle}{E_{0}(\lambda)-E_{n}(\lambda)} .
$$

A similar perturbation procedure can be applied to find the function $\left|\Phi^{i}\left(\lambda_{L}\right)\right\rangle$,

$$
\left|\Phi^{i}\left(\lambda_{L}\right)\right\rangle=\left|\Phi^{i}\left(\lambda_{R}\right)\right\rangle-\delta \lambda\left|\Phi^{i \prime}\left(\lambda_{R}\right)\right\rangle .
$$

Thus, for the function $\left|\Psi_{0}\left(\lambda_{L}\right)\right\rangle$ (see the first Eq. A15) we obtain

$$
\left|\Psi_{0}\left(\lambda_{L}\right)\right\rangle=\left|z_{i}\right\rangle \otimes\left|\Phi^{i}\left(\lambda_{R}\right)\right\rangle-\delta \lambda\left|z_{i}\right\rangle \otimes\left|\Phi^{i \prime}\left(\lambda_{R}\right)\right\rangle
$$

Comparing with (A17), taking into account (A15), to the zeroth order perturbation, we obtain

$$
\left|z_{i}\right\rangle \otimes\left|\Phi^{i}\left(\lambda_{R}\right)\right\rangle=\left|z_{j}\right\rangle \otimes\left|\Phi^{j}\left(\lambda_{R}\right)\right\rangle,
$$

which can be true if

$$
\begin{aligned}
\left|\Phi^{i}\left(\lambda_{R}\right)\right\rangle & =\left|z^{j}\right\rangle \otimes\left|\Phi^{i j}\left(\lambda_{R}\right)\right\rangle, \\
\left|\Phi^{j}\left(\lambda_{R}\right)\right\rangle & =\left|z^{i}\right\rangle \otimes\left|\Phi^{i j}\left(\lambda_{R}\right)\right\rangle,
\end{aligned}
$$

where $\left|\Phi^{i j}\left(\lambda_{R}\right)\right\rangle$ belongs to the Hilbert space $S_{i j}$ of all qubits except qubits $i$ and $j$. Therefore,

$$
\left|\Psi_{0}\left(\lambda_{R}\right)\right\rangle=\left|z_{i}\right\rangle \otimes\left|z_{j}\right\rangle \otimes\left|\Phi^{i j}\left(\lambda_{R}\right)\right\rangle
$$

Comparing with the first line in A15 it is clear that $\left\langle\sigma_{i}^{z}\right\rangle$ does not change from $\lambda_{L}$ to $\lambda_{R}$.

Looking back at all three cases, we see that for (i) and (iii), $\left\langle\sigma_{i}^{z}\right\rangle$ does not change with $\lambda$ as the theorem states, while (ii) contradicts with the conditions of the theorem, namely the non-degeneracy of the ground state. 


\section{Appendix B: Linear susceptibility}

To calculate susceptibility using the non-degenerate perturbation theory (at small external biases $h_{j}$ ) we write the Hamiltonian as $H=H_{0}-\sum_{j} h_{j} \sigma_{j}^{z}$, where $H_{0}$ is the unperturbed Hamiltonian with eigenstates $\left|\Psi_{n}\right\rangle$ and eigenvalues $E_{n}$. To the first order perturbation in $h_{j}$, the ground state of $H$ is given by

$$
\left|\tilde{\Psi}_{0}\right\rangle=\left|\Psi_{0}\right\rangle-\sum_{j} h_{j} \sum_{n>0} \frac{\left\langle\Psi_{n}\left|\sigma_{j}^{z}\right| \Psi_{0}\right\rangle}{E_{0}-E_{n}}\left|\Psi_{n}\right\rangle .
$$

One therefore obtains

$$
\begin{aligned}
\left\langle\tilde{\Psi}_{0}\left|\sigma_{i}^{z}\right| \tilde{\Psi}_{0}\right\rangle & =\left\langle\Psi_{0}\left|\sigma_{i}^{z}\right| \Psi_{0}\right\rangle \\
& -\sum_{j} h_{j} \sum_{n>0} \frac{\left\langle\Psi_{0}\left|\sigma_{j}^{z}\right| \Psi_{n}\right\rangle\left\langle\Psi_{n}\left|\sigma_{i}^{z}\right| \Psi_{0}\right\rangle}{E_{0}-E_{n}} \\
& -\sum_{j} h_{j} \sum_{n>0} \frac{\left\langle\Psi_{0}\left|\sigma_{i}^{z}\right| \Psi_{n}\right\rangle\left\langle\Psi_{n}\left|\sigma_{j}^{z}\right| \Psi_{0}\right\rangle}{E_{0}-E_{n}} .
\end{aligned}
$$

The cross-susceptibility can be written as

$$
\begin{aligned}
\chi_{i j} & =\frac{\partial\left\langle\sigma_{i}^{z}\right\rangle}{\partial h_{j}}=\sum_{n>0} \frac{\left\langle\Psi_{0}\left|\sigma_{j}^{z}\right| \Psi_{n}\right\rangle\left\langle\Psi_{n}\left|\sigma_{i}^{z}\right| \Psi_{0}\right\rangle}{E_{n}-E_{0}} \\
& +\sum_{n>0} \frac{\left\langle\Psi_{0}\left|\sigma_{i}^{z}\right| \Psi_{n}\right\rangle\left\langle\Psi_{n}\left|\sigma_{j}^{z}\right| \Psi_{0}\right\rangle}{E_{n}-E_{0}} .
\end{aligned}
$$

[1] O. Gühne and G. Tóth, Phys. Rep. 474, 1 (2009).

[2] L. Amico, R. Fazio, A. Osterloh, and V. Vedral, Rev. Mod. Phys. 80, 517 (2008).

[3] G. Vidal, Phys. Rev. Lett. 91, 147902 (2003).

[4] R. Jozsa and N. Linden, Proc. R. Soc. Lond. A 459, 2011 (2003).

[5] W.K. Wootters, Phys. Rev. Lett. 80, 2245.

[6] G. Vidal and R. F. Werner, Phys. Rev. A 65, 032314 (2002).

[7] D.A. Meyer and N.R. Wallach, J. Math. Phys. 43, 4273 (2002).

[8] G.K. Brennen, Quant. Inf. Comput. 3, 616 (2003).

[9] P.J. Love et al., Quant. Information Processing 6, 187 (2007).

[10] F.M. Spedalieri, Phys. Rev. A 86, 062311 (2012).

[11] M. Neeley et al., Nature 467, 570 (2010).

[12] L. DiCarlo et al., Nature 467, 574 (2010).

[13] M. Steffen et al., Science 313, 1423 (2006).

[14] D. Greenberger, M. Horne, A. Shimony, A. Zeilinger, Am. J. Phys. 58, 1131 (1990).

[15] M. Ansmann et al., Nature 461, 504 (2009).
[16] J.R. Friedman, V. Patel, W. Chen, S.K. Tolpygo, and J.E. Lukens, Nature 406, 43 (2000).

[17] A.J. Berkley et al., Science 300, 1548 (2003).

[18] A.J. Berkley et al., Phys. Rev. B 87, 020502(R) (2013).

[19] S. Ghosh, T.F. Rosenbaum, G. Aeppli, and S.N. Coppersmith, Nature 425, 48 (2003).

[20] M. Wiesniak, V. Vedral, and C. Brukner, New J. Phys. 7, 258 (2005).

[21] A.M. Souza, M.S. Reis, D.O. Soares-Pinto, I.S. Oliveira, and R.S. Sarthour, Phys. Rev. B 77, 104402 (2008).

[22] D.O. Soares-Pinto et al., Europhys. Lett. 87, 40008 (2009).

[23] C. Brukner, V. Vedral, and A. Zeilinger, Phys. Rev. A 73, 012110 (2006).

[24] R. Harris et al., Phys. Rev. B 82, 024511 (2010).

[25] M.W. Johnson et al., Nature 473, 194 (2011).

[26] J. Karthik, A. Sharma, and A. Lakshminarayan, Phys. Rev. A 75, 022304 (2007).

[27] J.H. Van Vleck, Rev. Mod. Phys. 50, 181 (1978). 